\title{
AUTOMATIC ANTI-GLARE SYSTEM FOR NIGHT TIME DRIVING USING LIQUID CRYSTAL SCREENS
}

\author{
Anupama V ${ }^{\mathbf{1}}$, Divya Pankaj ${ }^{2}$, Gayathri Anand ${ }^{3}$, Soman K P ${ }^{4}$ \\ ${ }^{I}$ Department of CEN, Amrita Vishwa Vidyapeetham, Tamil Nadu, India \\ ${ }^{2}$ Department of CEN, Amrita Vishwa Vidyapeetham, Tamil Nadu, India \\ ${ }^{3}$ Department of CEN, Amrita Vishwa Vidyapeetham, Tamil Nadu, India \\ ${ }^{4}$ Department of CEN, Amrita Vishwa Vidyapeetham, Tamil Nadu, India
}

\begin{abstract}
This paper proposes a prototype model for an automatic anti-glare system to reduce the risk of accidents during night time driving. The architecture consists of an aperture assembly and sensors. The aperture assembly is a two dimensional array of aperture elements, and their transmittances can be independently controllable. According to the transmittance, each aperture element will act either as opaque or transparent. The intensity of light ray is modulated by the transmittance of aperture elements, which causes the reduction of intensity of light passing through it. The prototype is implemented using liquid crystal display (LCD) and photovoltaic sensors. This system provides an automated mechanism to control the illumination according to the direction of incoming light. The fundamental idea is to measure the incoming light and using measured values to generate patterns on the LCD to reduce the illumination. The proposed prototype can be implemented in two ways: i) as spectacles with liquid crystal sheets placed in front of the glasses, which can be used while driving, ii) by placing large liquid crystal sheets in front of the windshields of vehicles. This method provides a flexible low cost implementation to build simple, reliable device to reduce the intensity of the light on-the-fly.
\end{abstract}

Keywords: LCD, glare, sensor, transmittance

\section{INTRODUCTION}

Night time driving had become a greater challenge as road accidents occur due to poor lighting conditions at that time. This rate is approximately three to four times higher than that of the day time [1]. The accidents can occur from the following facts- limited visibility due to low light conditions, temporarily blindness due to glare from the headlights of other vehicles tiredness. Driving at night is a difficult task even for experienced drivers as they need to see the traffic control devices, lane lines, vehicles, pedestrians, animals, and other hazards. Illuminating roadways using artificial lighting can be used as a countermeasure for this problem. But too much light or improper lighting may result in glare, causing visual discomfort and a diminished ability to see the environment [2], [3].

Glare is an important factor as it can cause temporary blindness, confusion and dizziness. It causes difficulty in vision at night in the presence of bright light such as car headlamps [4]. It can be classified into two types: discomfort glare and disability glare. Discomfort glare causes a desire to look away from a bright light source or difficulty in seeing an object. Disability glare limits the vision without causing discomfort. Although nearly all studies agree that glare from headlights results in discomfort glare for many drivers, glare can be tackled by following ways: i) reducing the intensity of the light source ii) reducing the illumination reaching the driver's eye iii) increasing the glare angle iv) indirectly minimizing the effects of glare. There are several ways to reduce and handle glare, but most of them are certain precautions a driver must take [5]. Examples of such precautions are clearing windshield windows and glass surfaces, cleaning vehicle's headlights, avoid looking directly at head lights etc. Glare can also be controlled by wearing polarized sunglasses [10]. But such types of glasses are hazardous since they give the driver a feeling of seeing better when in fact it is not like that.

By considering this situation the automotive engineers, designers, and researchers are trying to improve the safety measures for the next generation of automobiles. Currently, there are only two practical methods existing for night time lighting: fixed overhead lighting and vehicle headlights. Several studies are done on glare reduction during both day and night driving. Previous attempts include methods such as cut out glasses, pinhole glasses, special night-time cut-out flip down visors, headlight slats and even liquid products which reduce glare if applied to the windshield. All these methods have their own limitations. Aaron Shield and Rafael Cosman proposed a prototype for reducing glare due to sunlight using electrochromic panels [7]. This approach was limited only to day-time driving [11]. Torsten Steiner, Karsten Roscher and Josef Jiru proposed a cooperative automatic glare reduction 
mechanism using vehicle-to-vehicle radio technology [8]. But this mechanism will only help to reduce the glare due to headlights of vehicles. Another method which made a change to the glare reduction technology is CMOS (Complementary Metal Oxide Semiconductor) glare sensor [6] which communicates with microcontroller and in turn triggers to select pixels on a liquid crystal display and eliminates glare sources. This system is comprised of CMOS as sensor which makes the system more complex. Another disadvantage of this system is that, it requires large amount of processing.

In this paper, we propose a prototype using liquid crystal displays (LCD) to reduce the illumination reaching the driver's eye. Unlike other methods, this system provides an automated mechanism to control the illumination according to the direction of incoming light. The fundamental idea is to measure the incoming light and using measured values to generate patterns on the LCD to reduce the illumination. The proposed prototype can be implemented in two ways: i) as spectacles with liquid crystal sheets placed in front of the glasses, which can be used while driving, ii) by placing large liquid crystal sheets in front of the windshields of vehicles.

The paper is organized as follows. In the next section the architecture of work and the system prototype is described. Section 3 describes experimental setup and results.

\section{SYSTEM OVERVIEW}

\subsection{Description of System Architecture}

The proposed architecture consists of sensors and the aperture assembly, as shown in Figure 1. The aperture assembly is a two dimensional array of aperture elements and their transmittance can be individually controlled. The sensor is the light detection element used to measure the incoming light. A phototransistor or photodiode can be used as the sensor. Liquid Crystal Sheets are used as the aperture assembly and their size can be decided according to the type of implementation. The implementation as spectacles requires LCD sheets of small size while the windshield implantation requires flexible LCD sheets of larger size.

The lights from incoming vehicles are measured using the sensors and the transmittances of aperture elements are controlled based on the measured values. Hence the amount of light that passes through the aperture assembly is reduced, and consequently reducing the glare.

\subsection{Liquid Crystal Display (LCD)}

The LCD panels are the most common form of displays used in televisions, computers, mobile phones, watches etc. They are also used for image acquisition in lensless cameras proposed by Bell Labs [9].

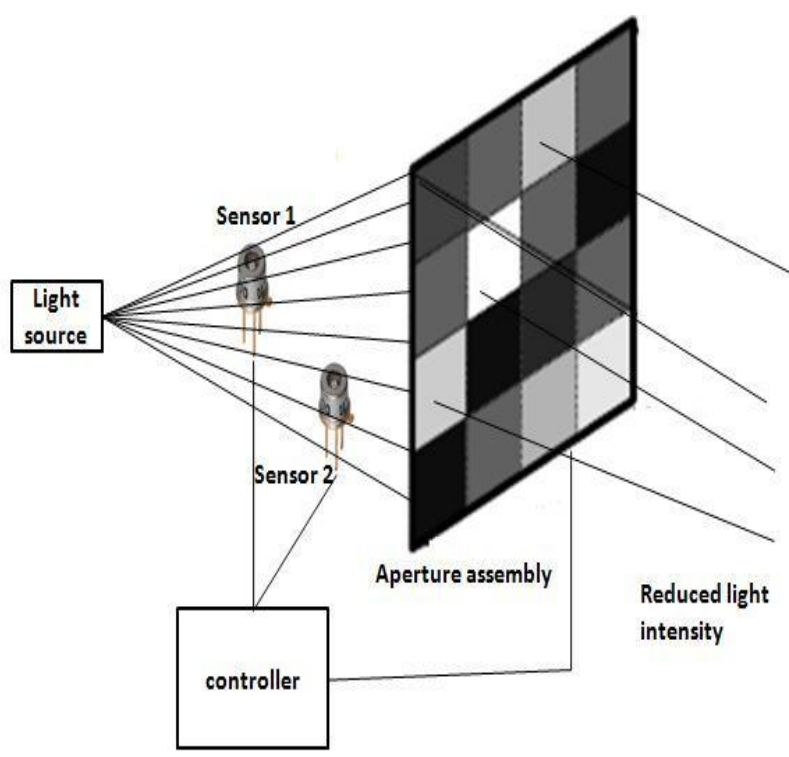

Fig -1: The proposed architecture

The proposed architecture makes use of LCD panels to reduce the intensity of light passing through it by making the liquid crystals clear or solid. The solid liquid crystals will block the light passing through it, thereby causes a significant reduction in the intensity of light passing through the panel.

\subsection{Proposed Method}

The system allows LCD aperture to transmit light in a controlled manner to reduce glare. A transmittance matrix $\mathrm{T}(\mathrm{x}$, y) is defined for the aperture assembly based on the measured light intensity. When transmittance of the aperture element is 1 , it will act as transparent and when transmittance is 0 , it will act as opaque. The transmittance matrix is given by

$$
\begin{array}{ccc}
T(x, y) \square & 0, & p(x, y) \text { is opaque } \\
1, & p(x, y) \text { is transparent }
\end{array}
$$

where $\mathrm{p}(\mathrm{x}, \mathrm{y})$ is the aperture element at position $(\mathrm{x}, \mathrm{y})$ in the aperture assembly. The transmittance for the aperture assembly will make the required aperture elements (the part of aperture assembly through which more light is passing) opaque by making their transmittance as 0 . The intensity of light coming from different directions can be measured using sensors. A threshold value, $t_{h}$ for light intensity is defined according to the application which is used to compare the sensor measurements.

Two sensors $S_{1}$ and $S_{2}$ are used to measure light coming from left and right direction. The light intensity measurement from the sensors $S 1$ and $S 2$ can be defined as $I_{1}$ and $I_{2}$ respectively. The controller will generate the transmittance matrix by comparing the sensor measurements with the threshold value. 
If $\mathrm{I}_{1}>\mathrm{t}_{\mathrm{h}}$, the transmittance matrix is defined such that the aperture elements corresponding to the left direction are made opaque. Similarly, if $I_{2}>t_{h}$, the aperture elements corresponding to the right direction are made opaque. The intensity of the light ray is modulated by the transmittance of aperture elements and thus reducing the intensity of light falling on the driver's eye.

The prototype can be implemented either in the form of spectacles or on the form of windshield of vehicles. The implementation in the form of spectacles and windshields are shown in Figure 2 and 3 respectively. The spectacles implementation uses LCD panels of small pixel size, whereas the windshield implementation used large and flexible LCD panels.

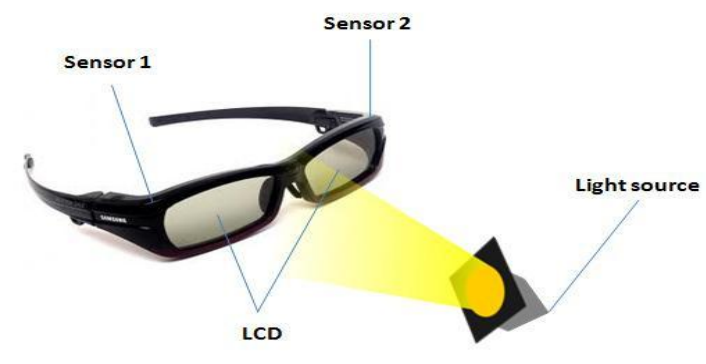

Fig -2: Spectacles implementation

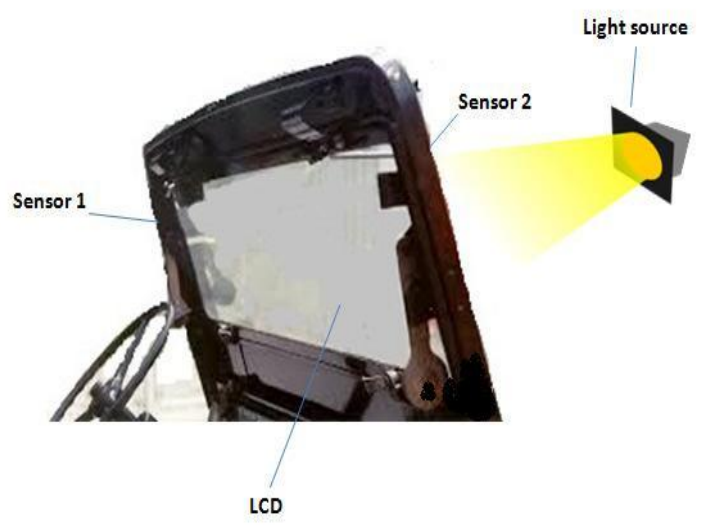

Fig -3: Windshield implementation

\section{EXPERIMENTAL SETUP AND RESULTS}

The experimental setup comprises of LCD, photovoltaic sensors and an arduino based controller. For testing spectacles type implementation is used. Aperture assembly is build using Nokia 5110 LCD through which light is passed. It contains $84 \times 48$ graphic LCD which runs at $3.3 \mathrm{~V}$ or $5 \mathrm{~V}$. The aperture elements are made opaque or transparent by generating random patterns programmatically on LCD. The intensity of incoming light can be measured using phototransistors placed in different directions. Phototransistors will sense the light and the measured light intensity values are transmitted to the controller. Arduino microcontroller is used to generate the patterns corresponding to the measured values. Arduino also provides the power required to run the LCD.

Results are obtained in two conditions: with and without the glare control. The values are obtained by measuring the intensity of light from different sources passed by the LCD the intensity values thus obtained are shown in Figure 4 and 5. From the results it can be observed that the intensity of light reduces significantly with glare control mechanism. The controller can reduce the light intensity by a greater amount and thus can cause the removal of glare. This shows that glare can be controlled on-the-fly.

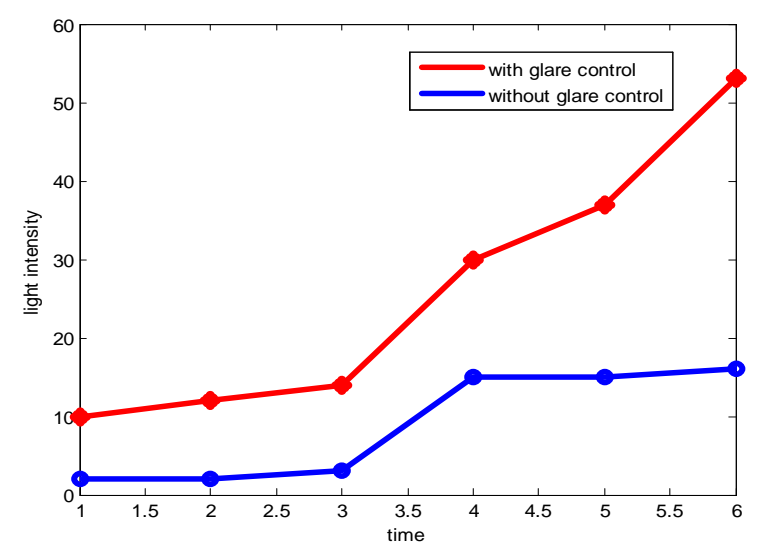

Fig -4: Variation in light intensity for light from a high intensity source with and without glare control

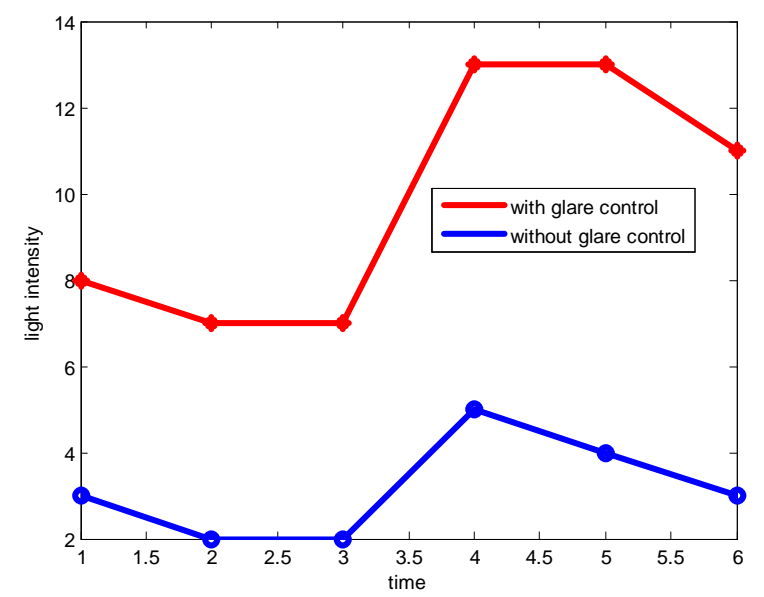

Fig -4: Variation in light intensity for light from a low intensity source with and without glare control 


\section{CONCLUSIONS}

A novel architecture for glare reduction is proposed. The proposed method provides a flexible low cost implementation to build simple, reliable device to reduce the intensity of the light on-the-fly. The implementation can be done in spectacles form or windshield form.

\section{REFERENCES}

[1]. S. Plainis, I. J. Murray, and I. G. Pallikaris, "Road traffic casualties: understanding the night-time death toll." Injury prevention: journal of the International Society for Child and Adolescent Injury Prevention, vol. 12, no. 2, pp. 125-8, April, 2006.

[2]. B. W"ordenweber, J. Wallaschek, P. Boyce, and D. D. Hoffman, "Automotive Lighting and Human Vision", 2007.

[3]. Schreuder, D. A, "Road Lighting for Safety." London: Thomas Telford Publishing. p. 107. ISBN 0-7277-2616-1. Retrieved September 25, 2009

[4]. Dr.Sanjay Dhawan, "Glare". Available: http://sdhawan.com/ophthalmology/glare

[5]. Mace, D., Garvey, P., Porter, R.J., Schwab, R., \& Adrian, W. "Countermeasures for Reducing the Effects of Headlight Glare." Washington, DC: AAA Foundation for Traffic Safety, 2001.

[6]. K. Bhagavathula, A. H. Titus, and C. Mullin, "An Extremely Low-Power CMOS Glare Sensor," IEEE Sensors Journal, vol. 7, pp. 1145-1151, 2007.

[7]. Sean Hollister.www.engadget.com. "Auto-dimming electrochromic panels reduce glare when driving", 2010.

[8]. T. Steiner, K. Roscher, J. Jiru. "Cooperative Glare Reduction Using V2V Radio Technology", 5th International Symposium on Wireless Vehicular Communications: WIVEC2013, Dresden, Germany, 2-3 June, 2013.

[9]. G. Huang, H. Jiang, K. Matthews and P. Wilford, "Lensless imaging by compressive sensing", IEEE International Conference on Image Processing, ICIP 2013, Sept, 2013.

[10]. Empire Optical Inc. "Polarized Lenses." Available :http://www.empireoptical.org/downloadable $\% 20$

files/reference/lens_info/Polarized\%20Lenses, 2012.

[11]. T. J. van den Berg, "On the relation between glare and straylight," Doc. Ophthalmol., vol. 78, no. 3-4, pp.177-181, 1991.

\section{BIOGRAPHIES}

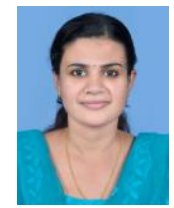

Anupama $\mathbf{V}$ is a Post graduate student in Amrita School of Engineering, Coimbatore, Tamil Nadu. Her qualifications include B.Tech. in Electronics and Communication Engineering in Dhanalakshmi Srinivasan Engineering College, Perambalur, Tamil Nadu, India. Her research interests include image processing and Embedded Systems

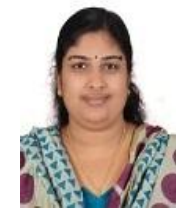

Divya Pankaj is a Post graduate student in Amrita School of Engineering, Coimbatore, Tamil Nadu. Her qualifications include B.Tech. in Electronics and Communication Engineering in Dhanalakshmi Srinivasan Engineering College, Perambalur, Tamil Nadu, India. Her research interests include image processing, optimization and Embedded Systems.

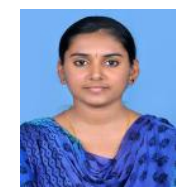

Gayathri Anand is a Post graduate student in Amrita School of Engineering, Coimbatore, Tamil Nadu. Her qualifications include B.Tech. in Electronics and Communication Engineering in College of Engineering and Management, Punnapra, Kerala, India. Her research interests include image processing, optimization and Embedded Systems.

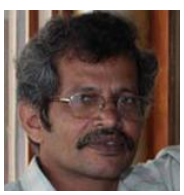

Dr. K.P Soman is the head, CEN, Amrita Vishwa Vidyapeetham, Ettimadai, Coimbatore64112. His qualifications include B.Sc. Engg. in Electrical engineering from REC, Calicut. P.M. Diploma in SQC and OR from ISI, Calcutta .M.Tech (Reliability engineering) from IIT, Kharagpur, $\mathrm{PhD}$ (Reliability engineering) from IIT, Kharagpur. Dr. Soman held the first rank and institute silver medal for M.Tech at IIT Kharagpur. His areas of research include optimization, data mining, signal and image processing, neural networks, support vector machines, cryptography and bio-informatics. He has over 55 papers in national and international journals and proceedings. He has conducted various technical workshops in India and abroad. 